\title{
Construction of Streptomyces lydicus A01 transformant with the chit33 gene from Trichoderma harzianum CECT2413 and its biocontrol effect on Fusaria
}

\author{
WU Qiong ${ }^{1}$, BAI LinQuan ${ }^{2}$, LIU WeiCheng ${ }^{3}$, LI Ying Ying ${ }^{1}$, LU CaiGe ${ }^{3}$, LI YaQian ${ }^{1}$, \\ LIN Zhen Ya ${ }^{1}$, WANG Meng ${ }^{1}$, XUE ChunSheng $^{4}$ \& CHEN Jie ${ }^{1 *}$ \\ ${ }^{1}$ School of Agriculture and Biology, Shanghai Jiao Tong University, Shanghai 200240, China; \\ ${ }^{2}$ School of Life Science and Biotechnology, Shanghai Jiao Tong University, Shanghai 200030, China; \\ ${ }^{3}$ Institute of Plant and Environment Protection, Beijing Academy of Agriculture and Forestry Sciences, Beijing 100097, China; \\ ${ }^{4}$ School of Plant Protection, Shenyang Agricultural University, Shenyang 110161, China
}

Received October 20, 2012; accepted March 7, 2013; published online May 31, 2013

\begin{abstract}
Streptomyces lydicus A01 resists many plant pathogens (including Fusarium spp.) by producing the antifungal agent natamycin, which binds to the ergosterol of fungal cell membranes and inhibits the growth of pathogens. Trichoderma harzianum CECT2413 is a widely-distributed soil fungus that antagonizes several plant fungal pathogens (including Fusarium spp.) by producing chitinase and degrading chitin, a major component of the fungal cell wall. This study attempted to enhance the biocontrol effect of $S$. lydicus A01 on Fusarium spp. by transforming the chitinase gene of Trichoderma. Chitinase and natamycin could act synergistically on both the cell walls and cell membranes of pathogens. The 33-kD chitinase-encoding gene (chit33) was cloned and conjugal-transformed from T. harzianum CECT2413 to S. lydicus A01, and then confirmed via polymerase chain reaction (PCR) assays. Subsequent analyses using the 3,5-dinitrosalicylic acid (DNS) method and ultraviolet spectrophotometry showed that compared with its wild type strain (WT), the S. lydicus A01 conjugal transformant (CT) with chit33 gene exhibited substantially higher chitinase activity and natamycin production. The resistance of S. lydicus A01-chit33 CT and WT to four Fusaria in crops and vegetables was tested via the cup-plate method. Compared with the WT, the conjugal transformant of S. lydicus A01 with chit33 gene from T. harzianum CECT2413 showed greatly increased biocontrol effect on fusarium disease. This study would be beneficial to the development of high-quality antifungal bio-agents for agricultural applications via the synergy between the previously non-existent and pre-existing functions achieved through heterogeneous gene transformation.
\end{abstract}

Streptomyces lydicus A01, chit33 conjugal transformant, wide-type strain, chitinase activity, natamycin production, Fusarium

Citation: Wu Q, Bai L Q, Liu W C, et al. Construction of Streptomyces lydicus A01 transformant with the chit33 gene from Trichoderma harzianum CECT2413 and its biocontrol effect on Fusaria. Chin Sci Bull, 2013, 58: 3266-3273, doi: 10.1007/s11434-013-5860-9

Plant diseases, such as fusarium wilt, stalk rot, and root rot, cause substantial losses in the yield and quality of crops and vegetables such as cotton, peas, corn, and cabbages $[1,2]$. Fusarium diseases are mainly caused by Fusarium spp., which includes Fusarium oxysporum f.sp. vasinfectum, Fusarium solani f.sp. pisi, Fusarium graminearum, and Fusarium oxysporum f.sp. conglutinans [3-6]. The management of Fusarium remains difficult and the cultivation

*Corresponding author (email: jiechen59@gmail.com) of disease-resistant varieties has achieved limited success. This result is partially due to the rapid mutation of pathogens. In addition, the application of chemicals, such as preplant soil fumigation with methyl bromide, is poisonous to humans [7]. Therefore, studies have focused on the prevention and biocontrol of fusarium diseases because of a need for high-quality antifungal agents.

Streptomyces lydicus A01 is a biocontrol agent that produces natamycin and is antagonistic to a variety of pathogens (including Fusarium spp.) [8]. Natamycin is an antibi- 
otic macrolide polyene commonly produced by Streptomyces spp., such as Streptomyces natalensis and Streptomyces chattanoogensis. Natamycin can specifically bind to the ergosterol in fungal cell membranes and then block fungal growth by inhibiting vacuole fusion $[9,10]$. Natamycin can resist Fusarium isolated from keratitis. As the only available anti-fungal medication approved by the American Food and Drug Administration, natamycin is not toxic to humans [11] and has long been used as a food additive [12,13].

Another important biocontrol agent, Trichoderma spp., has also been used on a number of crops and vegetables for the biocontrol of pathogens, including Fusarium spp. [14-16]. The cell wall-degrading enzymes produced by Trichoderma harzianum, such as chitinases (chit33), proteases (prb1), and $\beta$-glucanases (bgn13.1), have been described as antagonistic components against $F$. oxysporum [17]. Compared to its wide-type strain, the T. harzianum Rifai TM transformants that overexpress the chit36 gene can more efficiently inhibit $F$. oxysporum [18]. Chitinase can hydrolyze the chitin, a $\beta$-1, 4-linked polymer of $N$-acetyl glucosamine present in most fungi (including Fusarium spp.) as a major cell wall component [19].

Natamycin and chitinase, produced by Streptomyces and Trichoderma, respectively, are common biocontrol agents used in the biocontrol of fungal diseases. To date, only a few chitinases have been identified in Streptomyces spp., such as a $49-\mathrm{kD}$ chitinase from Streptomyces griseus HUT 6037, with no chitinase detected in S. lydicus A01 [20]. Previous studies have attempted to enhance the antifungal activity of Streptomyces against pathogens mainly by enhancing their production of antibiotics production using ultraviolet mutation, chemical mutagenesis, or intraspecific protoplast fusion [21,22]. No study has examined the combined biocontrol effects of natamycin and chitinase on specific plant diseases related to Fusarium spp. thus far. Combining the high chitinase expression of Trichoderma with the substantial natamycin production of Streptomyces via transformation is a worthwhile goal. In this study, an engineering strain that can express both chitinase and natamycin was constructed via the conjugal transformation of the chit33 gene from $T$. harzianum CECT2413 to S. lydicus A01. Chitinase activity and natamycin production were tested for both S. lydicus A01-chit33 CT and wild-type (WT) via the 3,5-dinitrosalicylic acid method (DNS) and ultraviolet spectrophotometry. The associated anti-Fusaria activities of S. lydicus A01-chit33 CT and WT were examined via the cup-plate method. The results of this study could provide insights into the development of high-quality anti-fungal bio-agents through heterogeneous gene transformation.

\section{Materials and methods}

\subsection{Plasmids and strains}

The pIB139 and pHZ1272 vectors were provided by BAI
LinQuan (School of Life Science and Biotechnology, Shanghai Jiao Tong University). The pIB139 vector, which carries an apramycin resistance cassette and the promoter region of the erythromycin resistance gene (ermE* promoter), could express exogenetic protein constitutively by inserting the cassette into the Streptomyces genome. The DEV intermediate vector was purchased from Generay Biotech Co., Ltd. (China).

The cloning host, Escherichia coli strain DH5a, was purchased from Takara (Japan) and the donor for conjugal transformation, ET12567 (pUZ8002), was provided by Linquan Bai. The recipient strain, S. lydicus A01, and the pathogens, F. graminearum, F. oxysporum Schl. f.sp. conglutinans, $F$. solani f.sp. pisi, and $F$. oxysporum f.sp. vasinfectum, were provided by Liu Weicheng (Institute of Plant and Environment Protection, Beijing Academy of Agriculture and Forestry Sciences).

\subsection{Synthesis and amplification of the sequences}

The chit33 gene of T. harzianum CECT2413 [23] was synthesized according to the partial codons of Streptomyces coelicolor for high expression in S. lydicus A01 (Generay, China). In addition, the DNA sequence of the melC1 signal peptide of Streptomyces was synthesized [24]. The terminator sequence of Streptomyces was polymerase chain reaction (PCR)-amplified from the pHZ1272 vector with Ter-F1 and Ter-R2 primers (Table 1). The PCR conditions were as follows: $95^{\circ} \mathrm{C}$ for $10 \mathrm{~min}$, followed by 30 cycles at $95^{\circ} \mathrm{C}$ for $1 \mathrm{~min}, 55^{\circ} \mathrm{C}$ for $1 \mathrm{~min}$, and $72^{\circ} \mathrm{C}$ for $1 \mathrm{~min}$, and finally, $72^{\circ} \mathrm{C}$ for $10 \mathrm{~min}$.

The DNA sequence of the melC1 signal peptide was 5'CGGGGTCGTCAACCCAACGCACCCCAGGAGGTCCC GCATGCCGGAACTCACCCGTCGTCGCGCGCTCGGC GC-3'. The sequence of the terminator was 5'-AAAGTTTTG-TC-GTCTTTCCAGACGTTAGTAAATGAATTTTCT GTATGAGGTTTTGCTAAACAACTTTCAACAGTTTCA GCGGAGTGAGAATAGAAAGGAACAACTAAAGGAA TTGCGAATAATAATTTTTTCACGTTGAAAATCTCCA AAAAAAAAGGCTCCAAAAGGAGCCTTTAATTGTAT CGGTTTATCAGCTTGCTTTCGAGGTGAATTTCTTAA ACAGCTTGATACCGATAGTTGCGCCGACAATGACA ACAACCATCGCCCACGCATAACCGATATATTCGGT CGCTGAGGCTTGCAGGGAGTCAAAGGCCGCTTTTG CGGGATCTCGTCGAAGGCGGCGGGGGCGCCGGAC GCGGCCGGGTTCCCGGG-3'.

Table 1 The primers used in this study

\begin{tabular}{ll}
\hline Primer name & Primer sequence $\left(5^{\prime} \rightarrow 3^{\prime}\right)$ \\
\hline Ter-F1 & AAAGATATCCCCAAGCTTAAAGTTTTGTCGTCT \\
Ter-R2 & CCGGAATTCCCCGGGAACCCGGCCGCG \\
Apr-F1 & CGTCAACTCCAAGCAGAACA \\
Apr-R2 & CTCGAAGTCGAAGTCGAAGC \\
Chit33-F1 & GCTCATCGGTCAGCTTCTCA \\
Chit33-R2 & TCGCATTCTTCGCATCCC \\
\hline
\end{tabular}




\subsection{Gene cloning and conjugal transformation}

The construction procedure of the positive pIB139 vector was plotted into four cycles using Winplas 2.7 (Figure 1). The positive pIB139 vector was identified via digestion with Nde I and EcoR I, transformed to ET12567 (pUZ8002) for conjugal transformation to S. lydicus A01, and then analyzed using PCR assays. A blank pIB139 vector was transformed to verify whether it can affect chitinase activity and natamycin production without the chit33 gene. The conjugal transformation was performed according to Kieser et al. [25]. The genomes were extracted using a G+ bacteria genomic DNA kit (Zoman, China). Two pairs of primers were used to screen the putative ex-conjugants, that is, $S$. lydicus A01-chit33 CT via PCR assays, including S. lydicus A01 conjugal transformant with a blank pIB139 vector (S. lydicus A01-pIB139 CT). Chit33-F1 and Chit33-R2 were used to amplify part of the chit33 gene. Apr-F1 and Apr-R2 were used to amplify part of the apramycin-resistant gene in pIB139 (Table 1). This amplification was conducted under the following conditions: $95^{\circ} \mathrm{C}$ for $10 \mathrm{~min} ; 30$ cycles at $95^{\circ} \mathrm{C}$ for $1 \mathrm{~min}, 55^{\circ} \mathrm{C}$ for $1 \mathrm{~min}$, and $72^{\circ} \mathrm{C}$ for $1 \mathrm{~min}$; and $72^{\circ} \mathrm{C}$ for $10 \mathrm{~min}$.

\subsection{Chitinase activity assay}

The chitinase activity assay was performed with the help of a chitinase-inductive broth consisting of the seed broth $(0.5 \%$ glucose, $0.5 \%$ tryptone, $0.5 \%$ yeast extract, $0.05 \%$ $\mathrm{K}_{2} \mathrm{HPO}_{4}, 0.05 \% \mathrm{MgSO}_{4} \cdot 7 \mathrm{H}_{2} \mathrm{O}, 0.05 \% \mathrm{KCl}$, and $0.001 \%$ $\left.\mathrm{FeSO}_{4} \cdot 7 \mathrm{H}_{2} \mathrm{O}\right)$ and the fermentation broth $(1 \%$ chitin powder, $0.4 \% \mathrm{NH}_{4} \mathrm{NO}_{3}, 0.05 \% \mathrm{~K}_{2} \mathrm{HPO}_{4}, 0.05 \% \mathrm{MgSO}_{4} \cdot 7 \mathrm{H}_{2} \mathrm{O}$, $0.05 \% \mathrm{KCl}$, and $0.001 \% \mathrm{FeSO}_{4} \cdot 7 \mathrm{H}_{2} \mathrm{O}$ ). Briefly, the mycelia of S. lydicus A01-chit33 CT and WT (including S. lydicus A01-pIB139 CT) were inoculated to the seed broth, which was then incubated on a $180 \mathrm{r} \mathrm{min}^{-1}$ rotary shaker at $28^{\circ} \mathrm{C}$ for $2 \mathrm{~d}$. The precipitation of the seeds $(100 \mu \mathrm{L})$ was subsequently added to the fermentation broth, and the supernatant was obtained via centrifugation every two days from Days 2 to 14 . Chitinase activity was determined using the DNS method [26]. The $N$-acetyl- $D$-glucosamine (NAG) standard curve was prepared according to Reissig et al. [27]. One unit (U) of chitinase activity was defined as the amount of enzyme required to release $1 \mu \mathrm{g}$ of $N$-acetyl- $\beta$-glucosamine per hour.

Chitin-hydrolysis capability (diameter of the hydrolysis zone) was examined for the supernatants of the 10-d old fermentation broth of S. lydicus A01-chit33 CT and WT. Briefly, $200 \mu \mathrm{L}$ of the supernatant was inoculated to each Oxford cup on chitin-containing agar plates $(0.3 \%$ colloidal chitin, $0.4 \% \quad \mathrm{NH}_{4} \mathrm{NO}_{3}, 0.05 \% \quad \mathrm{~K}_{2} \mathrm{HPO}_{4}, 0.05 \% \mathrm{MgSO}_{4} \cdot$ $7 \mathrm{H}_{2} \mathrm{O}, 0.05 \% \mathrm{KCl}, 0.001 \% \mathrm{FeSO}_{4} \cdot 7 \mathrm{H}_{2} \mathrm{O}$, and $2 \%$ agar powder). The plates were incubated at $37^{\circ} \mathrm{C}$ in the dark, and the diameter of the chitin-hydrolysis zone was measured after $12 \mathrm{~h}$. Each measurement was performed at least in triplicate.

\subsection{Natamycin production assay}

Natamycin (Aladdin, China) solutions in methanol with different concentrations were measured at $A_{303}$ using ultraviolet spectrophotometry and a standard curve was prepared. Natamycin production was then determined according to the standard curve by measuring the absorbance of the fermentation supernatant dilution $(1: 10, \mathrm{v} / \mathrm{v}$, diluted in the methanol) of 2-d-, 6-d-, and 10-d-old S. lydicus A01-chit33 CT and WT (including S. lydicus A01-pIB139 CT) in the chitinase-inductive broth at $303 \mathrm{~nm}$ [8]. Each measurement was performed at least in triplicate.

\subsection{Inhibition test of S. lydicus for biocontrol of Fusaria}

Exactly $200 \mu \mathrm{L}$ of the fermentation supernatants of 10-d old S. lydicus A01-chit33 CT and WT in chitinase-inductive broth were added to two separate Oxford cups on the plates containing $25 \mathrm{~mL}$ of potato dextrose agar (PDA) supplemented with Fusaria spore suspension (final concentration $\left.10^{6} \mathrm{cfu} \mathrm{mL}^{-1}\right)$. The plates were incubated at $28^{\circ} \mathrm{C}$ for $2 \mathrm{~d}$,

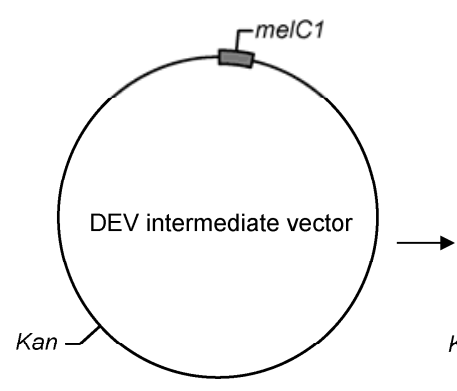

(a)

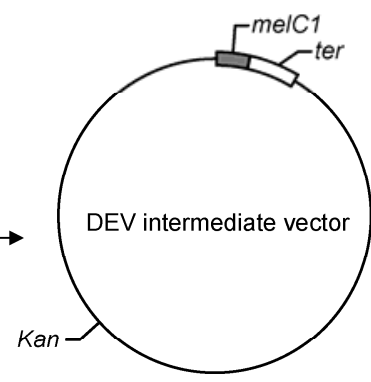

(b)

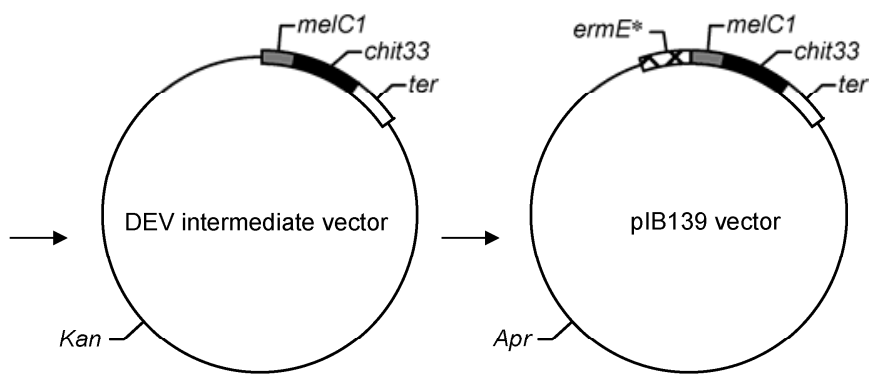

(c)

(d)

Figure 1 Cloning diagram of the chit33 gene. (a) The DNA sequence encoding the melC1 signal peptide inserted into the DEV intermediate vector after digestion with Nde I and BamH I; (b) Terminator sequence ligated to the signal sequence in the vector after digestion with EcoR V and EcoR I; (c) Synthesized chit33 gene inserted between the signal sequence and the terminator after digestion with BamH I and Hind III; (d) Fragment containing the DNA sequence of melC1 signal peptide, chit33 gene, and terminator gene excised from the DEV vector and inserted after the ermE* promoter in the pIB139 vector after digestion with $N d e$ I and EcoR I. 
and the diameter of the Fusaria inhibition zone was measured for S. lydicus A01-chit33 CT and WT. Each measurement was performed at least in triplicate.

\section{Results}

\subsection{Conjugal transformation of the chit33 gene into S. lydicus A01}

The sequence similarity (grey area) and difference (white area) of the chit33 gene before and after optimization were $81.7 \%$ and $18.3 \%$, respectively (Figure 2). The positive pIB139 vector containing chit33 was identified via digestion with Nde I and EcoR I. The pIB139 vector sequence was 5922 bp in length, and those of the melC1 signal peptide, chit33 gene, and the terminator were $1438 \mathrm{bp}$. The results were in agreement with the predetermination values (Figure 3). The S. lydicus A01-chit33 CT was identified via PCR assay (including S. lydicus A01-pIB139 CT). The theoretical lengths of the apramycin and chit33 genes amplicons in the pIB139 vector were 728 and 436 bp, respectively. The practical amplified results corresponded to the theoretical values. The pIB139 vector containing the chit33 gene (including the blank pIB139 vector) was inserted into the $S$. lydicus A01 genome, and engineering strains were successfully constructed (Figure 4).

\subsection{Chitinase activity of S. lydicus A01}

Compared with its WT, S. lydicus A01-chit33 CT displayed higher chitinase activity from Days 4 to 14 during fermentation, particularly from Days 10 to 14 during the stationary phase. On Day 10, the chitinase activity of S. lydicus A01chit33 CT was $452.664 \pm 30.537 \mathrm{U} \mathrm{mL}^{-1}$, which was 7.3 times that of the WT $\left(61.921 \pm 14.927 \mathrm{U} \mathrm{mL}^{-1}\right)$. The chitinase activity of $S$. lydicus A01-pIB139 CT was $65.656 \pm$ 13.364 $\mathrm{U} \mathrm{mL}^{-1}$ on Day 10, comparable to that of WT (Figure 5(a)). The heterogenous gene chit33 from T. harzianum CECT2413 was highly expressed, and the insertion of the pIB139 vector into the genome had nearly no impact on the chitinase expression of $S$. lydicus A01.

Further comparison of the chitin-hydrolysis haloes were only made between the fermentation supernatants of the $S$. lydicus A01-chit33 CT and WT on Day 10. The diameter of the hydrolysis zone produced by the fermentation supernatant of S. lydicus A01-chit33 CT averaged $1.3 \pm 0.1 \mathrm{~cm}$, substantially larger than that of $S$. lydicus A01 WT (average $0.4 \pm 0.1 \mathrm{~cm}$, Figure 5(b)). The results of the chitin-hydrolysis haloe analysis were consistent with those of the chitinase activity analysis via DNS method.

\subsection{Natamycin production of S. lydicus A01}

The natamycin production of S. lydicus A01-chit33 CT and WT (including S. lydicus A01-pIB139 CT) were examined at three time points. The natamycin production of $S$. lydicus A01-chit33 CT on Days 2, 6, and 10 were $1.677 \pm 0.706$, $18.182 \pm 1.161$, and $40.576 \pm 2.817 \mu \mathrm{g} \mathrm{mL}^{-1}$, respectively, and those of WT were $1.424 \pm 0.517,2.758 \pm 0.911$, and $5.970 \pm 1.318 \mu \mathrm{g} \mathrm{mL}^{-1}$, respectively. The natamycin production of S. lydicus A01-chit33 CT was 6.8 times that of the WT on Day 10, both proportional to chitinase activity in S. lydicus A01-chit33 CT and WT. By contrast, the natamycin production of $S$. lydicus A01-pIB139 CT on Days 2, 6, and 10 were $1.364 \pm 0.833,2.697 \pm 0.964$ and $5.818 \pm$ $1.289 \mu \mathrm{g} \mathrm{mL}^{-1}$, respectively, with no substantial differences from those of the WT (Figure 6).

Thus, the pIB139 vector had nearly no effect on the

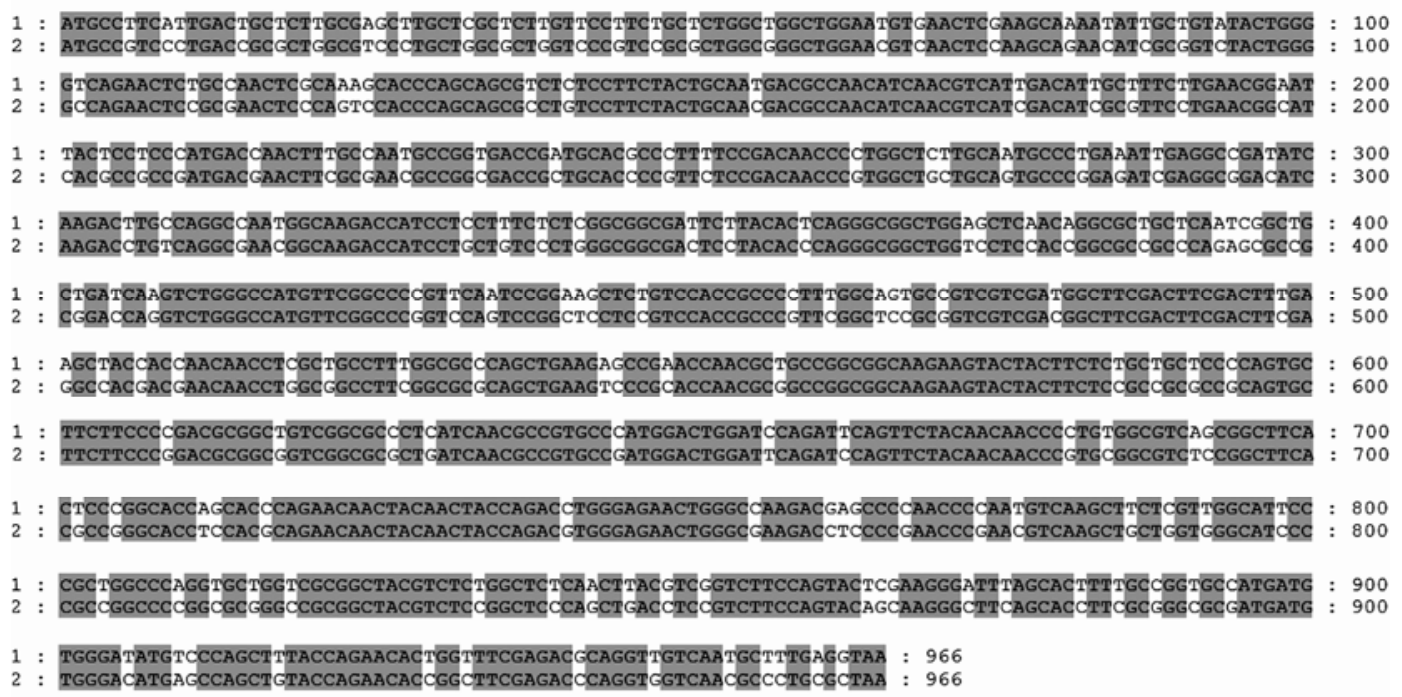

Figure 2 BLAST analysis of chit33 gene from T. harzianum CECT2413 and optimization according to the codon preferred by S. coelicolor. 1, Original chit33 gene from T. harzianum CECT2413; 2, The optimized chit33. 


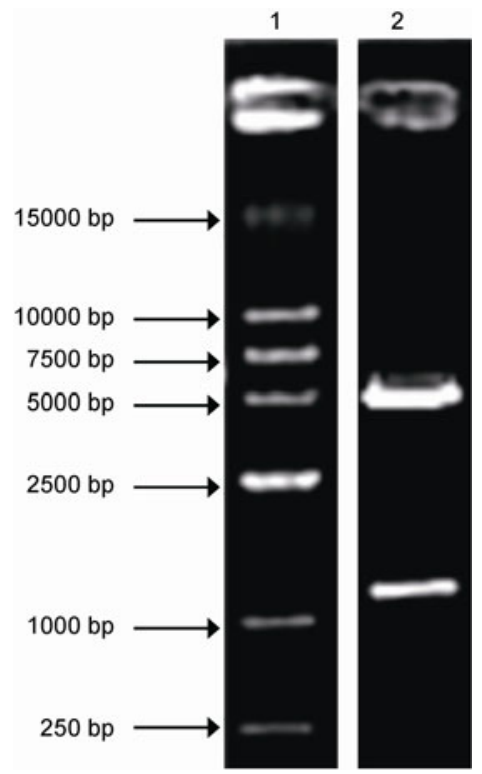

Figure 3 Gel image of positive pIB139 vector via double-digestion. 1, Marker DL15000 (TaKaRa, Japan); 2, positive pIB139 after double-digestion.

natamycin production of S. lydicus A01. In fact, natamycin production increased substantially rather than decreased after the transformation of the chit 33 gene.

\subsection{Biocontrol effect of S. lydicus A01 against Fusaria}

The diameters of the Fusaria inhibition zone produced by the 10-d-old fermentation supernatant of S. lydicus A01chit33 CT and WT were $1.7-1.8 \mathrm{~cm}$ and $0.35-0.8 \mathrm{~cm}$, respectively. The biocontrol effect of the fermentation supernatant produced by S. lydicus A01-chit33 CT on Fusaria was at least twice that of the WT (Figure 7). These results indicate that the inhibition effect of S. lydicus A01-chit33 CT on Fusaria was substantially greater than that of $S$. lydicus A01 WT.

\section{Discussion}

This study attempted to improve the biocontrol effect of $S$. lydicus A01 against Fusarium spp. via the conjugal transformation of chit33 from T. harzianum CECT2413. The in vitro tests showed that chitinase expression was substantially increased via the optimization of codon, the use of signal peptide, and the deployment of the ermE* strong promoter. The associated natamycin production of S. lydicus A01-chit33 CT was also substantially improved. These results suggest the possibility of synergizing the natamycin production of S. lydicus A01 and the chitinase activity of Trichoderma through chit33 gene transformation. In addition, a blank pIB139 vector was transformed to S. lydicus A01 in order to determine whether it affected the chitinase expression and natamycin production without the chit33 gene. Because S. lydicus A01-pIB139 CT and WT are little different in terms of chitinase activity and natamycin production, we inferred that the pIB139 vector had no impact. Therefore, the S. lydicus A01-pIB139 CT was no longer analyzed and only S. lydicus A01-chit33 CT and WT were subsequently discussed.

In this study, the induction broth used for the chitinase

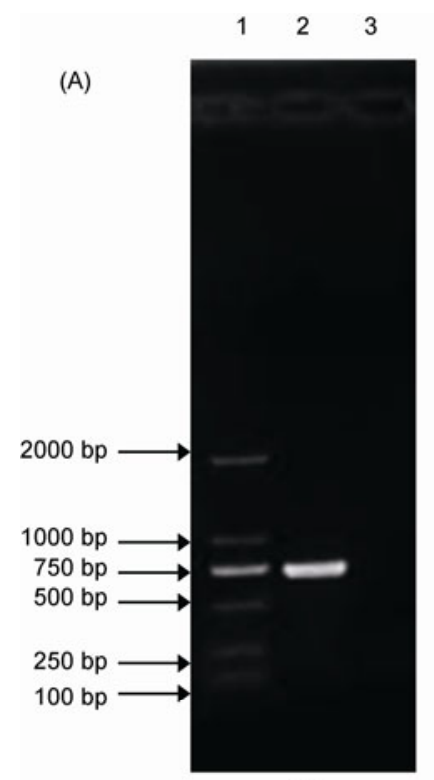

A1

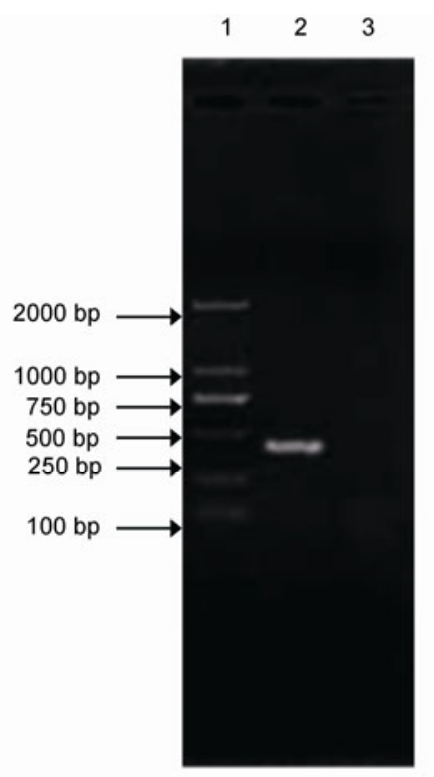

A2

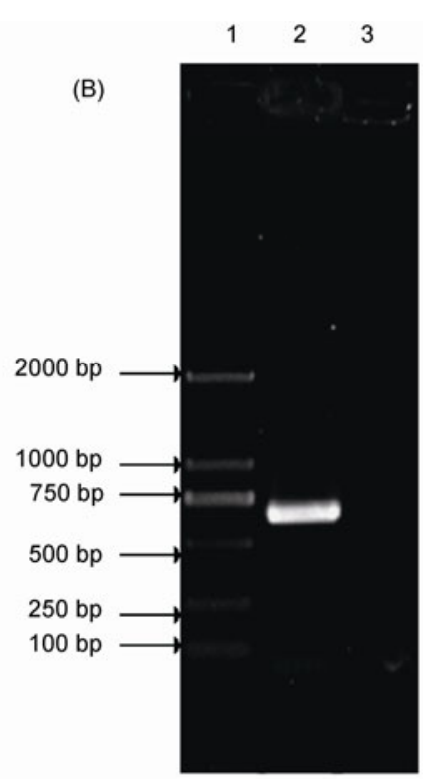

Figure 4 Gel image of S. lydicus A01-chit33 CT and S. lydicus A01-pIB139 CT via PCR assay. A1, Apramycin-resistant gene amplification: 1, Marker DL2000 (TaKaRa, Japan); A2, S. lydicus A01-chit33 CT; 3, S. lydicus A01 WT. A2, Chit33 gene amplification: 1, marker DL2000; 2, S. lydicus A01-chit33 CT; 3, S. lydicus A01 WT. (B) Apramycin-resistant gene amplification: 1, marker DL2000; 2, S. lydicus A01-pIB139 CT; 3, S. lydicus A01 WT. 


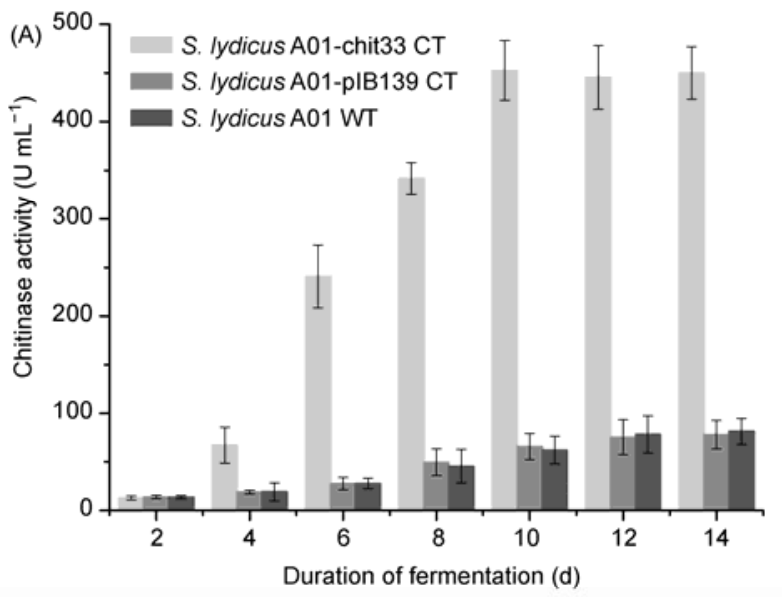

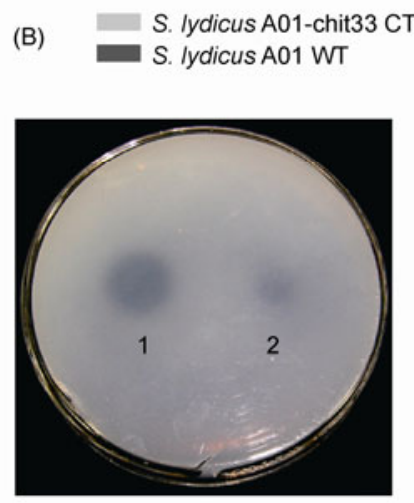

B1

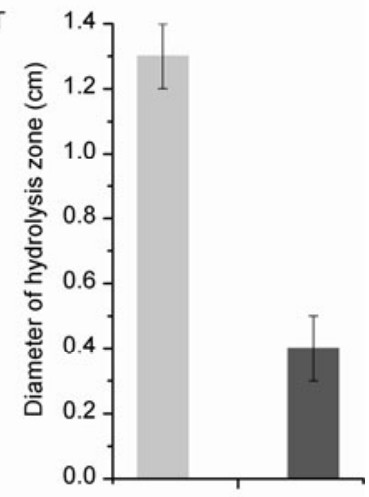

B2
Figure 5 Chitinase activity analysis. (A) Chitinase activities of S. lydicus A01-chit33 CT, S. lydicus A01-pIB139 CT, and WT; B1, Diameters of chitin-hydrolysis-haloes produced by $S$. lydicus A01-chit 33 CT and WT. 1, S. lydicus A01-chit33 CT; and 2, S. lydicus A01 WT; and B2, Statistic analysis of chitin-hydrolysis-haloes.

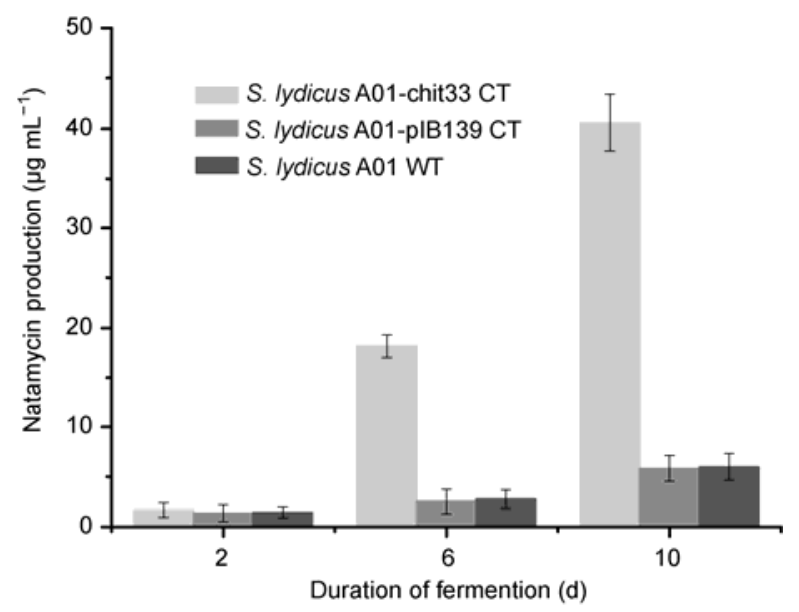

Figure 6 Natamycin productions of S. lydicus A01-chit33 CT, S. lydicus A01-pIB139 CT and WT.

activity assay contained chitin powder as the sole carbon source. This chitin powder stimulated the chitinase expression of S. lydicus A01-chit33 CT. Although most fungi or bacteria use colloidal chitin as the carbon source because they may not efficiently use chitin powder (e.g., Trichoderma harzianum TUBF 781, Trichoderma koningi with chit42 of Metarhizium anisopliae, and Streptomyces sp. M-20), S. lydicus A01-chit33 CT metabolized chitin powder with high efficiency [28-30]. Therefore, this engineering strain can be used in plant disease control and industrial chitin degradation.

The chitinase activity of both $S$. lydicus A01-chit33 CT and WT increased over time during the 10-d fermentation, and the associated natamycin production increased correspondingly. Owing to the high over-expression of chit33, the chitinase activity of $S$. lydicus A01-chit33 CT was substantially higher, consistent with the increase in natamycin production (Figures 5(a) and 6). Therefore, we hypothesize that chitinase degraded chitin into glucose, which was further absorbed and utilized by S. lydicus A01 for cell growth and natamycin production. Because the S. lydicus A01chit33 CT expressed much more chitinase than its WT, the former could grow faster and thus produce more natamycin.

The anti-Fusaria effect of the S. lydicus A01-chit33 CT was at least twice that of the WT via the synergy of natamycin and chitinase (Figure 7). However, natamycin and chitinase production level barely met the needs of agriculture. The associated culture broth needs to be improved by supplementing yeast extract powder and/or tryptone as carbon and nitrogen sources, except for the chitin powder and ammonium nitrate, to improve the chitinase activity and natamycin production of $S$. lydicus A01-chit33 CT. The broth $\mathrm{pH}$, incubation temperature, and rotation speed should also be optimized [28,31].

Because of their strong secretory system, Streptomyces can express several exogenous genes from fungi, animals, and humans, such as interferon- $\alpha 1$ and interleukin- 2 in humans expressed in Streptomyces lividens [32,33]. However, further study is needed to examine whether multiple functional genes can be transformed into S. lydicus A01 as novel high-quality biocontrol agents.

It is of importance to ensure that S. lydicus A01-chit33 CT has no environmental pollution or disruption effects based on environmental toxicity tests. Our future studies include in vitro experiments with vegetables and crops to solve more practical problems.

\section{Conclusions}

In this study, a conjugal transformant of S. lydicus A01 was constructed using the chit33 gene from T. harzianum CECT2413. As a result, the chitinase was highly expressed in S. lydicus A01-chit33 CT, and the associated natamycin production was substantially increased. Our results show that the fermentation supernatant produced by $S$. lydicus A01-chit33 CT had a much stronger biocontrol effect on Fusaria in crops and vegetables in the PDA plate, compared with the supernatant produced by the WT. To our knowledge, 
(A)

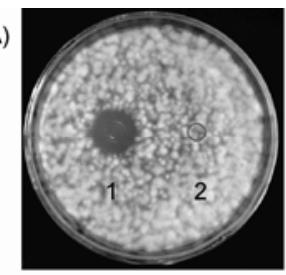

a

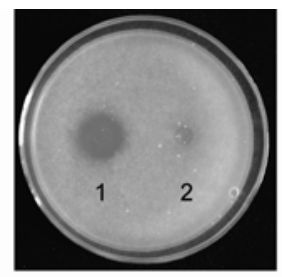

b

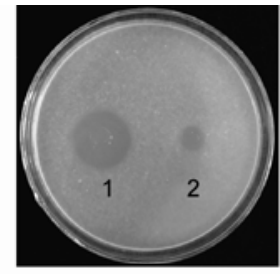

c

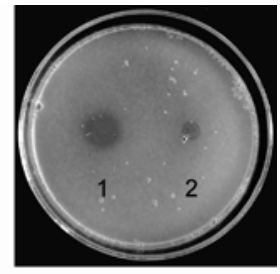

d

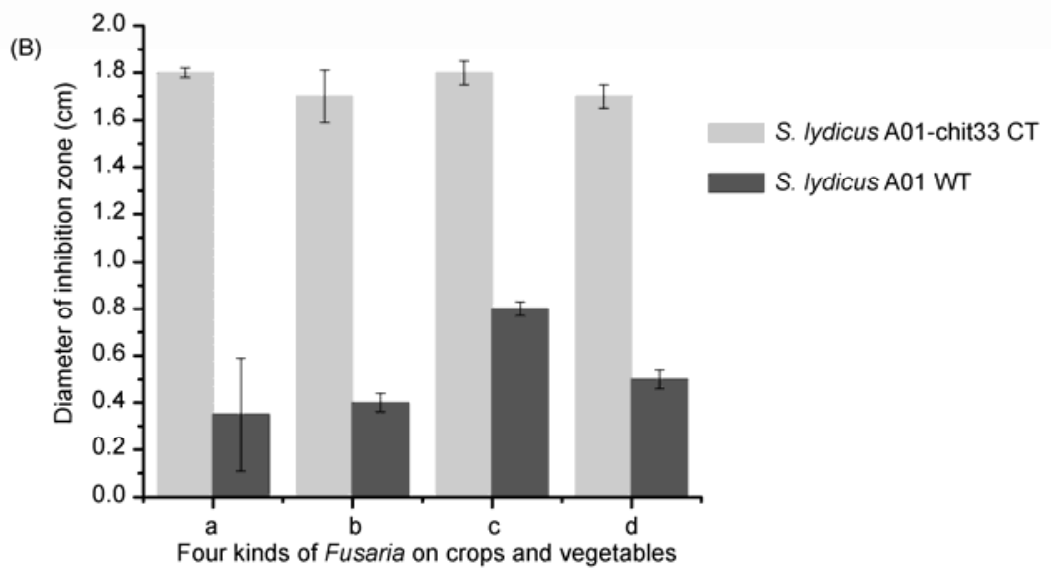

Figure 7 Control effects of S. lydicus A01-chit33 CT and WT against Fusaria. (A) Photos showing the control effects of S. lydicus A01-chit33 CT and WT against 4 kinds of Fusarium; a, Fusarium graminearum; b, Fusarium oxysporum Schl. f.sp. conglutinans; c, Fusarium solani f.sp. pisi; and d, Fusarium oxysporum f.sp. vasinfectum; 1, inhibition zones produced by S. lydicus A01-chit33 CT; and 2, inhibition zones produced by S. lydicus A01 WT. (B) Statistic analysis of the inhibition zones.

this study is first to present evidence for the improvement of the biocontrol effect of Streptomyces on Fusaria via the synergy of natamycin and chitinase effects. These findings could significantly affect the research and development of high-quality biocontrol agents through genetic transformation.

This work was supported by the Shanghai Municipal Science and Technology Commission (09391910900), the National Department Public Benefit Research Foundation of China (200903052), and the National High Technology Research and Development Program of China (2011AA10A205).

1 Sobowale A A, Odebode A C, Cardwell K F, et al. Antagonistic potential of Trichoderma longibrachiatum and T. hamatum resident on maize (Zea mays) plant against Fusarium verticillioides (Nirenberg) isolated from rotting maize stem. Arch Phytopathol Plant Prot, 2010, 43: 744-753

2 Chakrabarti A, Rep M, Wang B, et al. Variation in potential effector genes distinguishing Australian and non-Australian isolates of the cotton wilt pathogen Fusarium oxysporum f.sp. vasinfectum. Plant Pathol, 2011, 60: 232-243

3 Beckman C H, Roberts E M. On the nature and genetic basis for resistance and tolerance to fungal wilt diseases of plants. Adv Bo Res, 1995, 21: 35-77

4 Crawford M S, Kolattukudy P E. Pectate lyase from Fusarium solani f.sp. pisi: Purification, characterization, in vitro translation of the mRNA, and involvement in pathogenicity. Arch Biochem Biophys, 1987, 258: 196-205

5 Kommendahl T, Windels C E. Root-, stalk-, and ear-infecting Fusarium species on corn in the USA. In: Nelson P E, Toussoun T A, Cook R J, ed. Fusarium Diseases, Biology and Taxonomy. University
Park: The Pennsylvania State University Press, 1981. 94-103

6 Someya N, Tsuchiya K, Yoshida T, et al. Combined application of Pseudomonas fluorescens strain LRB3W1 with a low dosage of benomyl for control of cabbage yellows caused by Fusarium oxysporum f.sp. Conglutinans. Biocontrol Sci Tech, 2007, 17: 21-31

7 Fravel D, Olivain C, Alabouvette C. Fusarium oxysporum and its biocontrol. New Phytol, 2003, 157: 493-502

8 Lu C G, Liu W C, Qiu J Y, et al. Identification of an antifungal metabolite produced by a potential biocontrol Actinomyces strain A01. Braz J Micro, 2008, 39: 701-707

9 te Welscher Y M, ten Napel H H, Balagué M M, et al. Natamycin blocks fungal growth by binding specifically to ergosterol without permeabilizing the membrane. J Biol Chem, 2008, 283: 6393-6401

10 te Welscher Y M, Jones L, van Leeuwen R M, et al. Natamycin inhibits vacuole fusion at the priming phase via a specific interaction with ergosterol. Antimicrob Agents Ch, 2010, 54: 2618-2625

11 Lalitha P, Shapiro B L, Loh A R, et al. Amphotericin B and natamycin are not synergistic in vitro against Fusarium and Aspergillus spp. isolated from keratitis. Br J Ophthalmol, 2011, 95: 744-745

12 de Ruig W G, van den Berg G. Influence of the fungicdes sorbate and natamycin in cheese coatings on the quality of the cheese. Neth Milk Dairy J, 1985, 39: 165-169

13 Pintado C M B S, Ferreira M A S S, Sousa I. Control of pathogenic and spoilage microorganisms from cheese surface by whey protein films containing malic acid, nisin and natamycin. Food Control, 2010, 21: $240-246$

14 Benítez T, Rincón A M, Limón M C, et al. Biocontrol mechanisms of Trichoderma strains. Int Microbiol, 2004, 7: 249-260

15 Segarra G, Casanova E, Avilés M, et al. Trichoderma asperellum strain T34 controls Fusarium Wilt disease in tomato plants in soilless culture through competition for iron. Microb Ecol, 2010, 59: 141149

16 Shashi K, Ashish J, Shukla D N. Antagonistic Effect of Trichoderma strains against Fusarium oxysporum f.sp. udum buttler causing Wilt of Pigeon Pea. Agric Sci Dig, 2010, 30: 189-191 
17 Lopez-Mondejar R, Ros M, Pascual J A. Mycoparasitism-related genes expression of Trichoderma harzianum isolates to evaluate their efficacy as biological control agent. Biol Control, 2011, 56: 59-66

18 Viterbo A, Haran S, Friesem D, et al. Antifungal activity of a novel endochitinase gene (chit36) from Trichoderma harzianum Rifai TM. FEMS Microbiol Lett, 2001, 200: 169-174

19 Kimoto H, Akamatsu M, Fujii Y, et al. Discoidin domain of chitosanase is required for binding to the fungal cell wall. J Mol Microb Biotech, 2010, 18: 14-23

20 Tanabea T, Kawaseb T, Watanabeb T, et al. Purification and characterization of a 49-kDa chitinase from Streptomyces griseus HUT 6037. J Biosci Bioeng, 2000, 89: 27-32

21 Chen Z, Wen J, Song Y, Wen Y, et al. Enhancement and selective production of avermectin B by recombinants of Streptomyces avermitilis via intraspecific protoplast fusion. Chin Sci Bull, 2007, 52: 616-622

22 Wang X J, Wang X C, Xiang W S. Improvement of milbemycin-producing Streptomyces bingchenggensis by rational screening of ultraviolet-and chemically induced mutants. World J Microb Biot, 2009, 25: 1051-1056

23 Limon M C, Lora J M, Garcfa I, et al. Primary structure and expression pattern of the 33-kDa chitinasegene from the mycoparasitic fungus Trichoderma harzianum. Curr Genet, 1995, 28: 478-483

24 Hong B, Wu B Y, Li Y. Production of C-terminal amidated recombinant salmon calcitonin in Streptomyces lividans. Appl Biochem Biotech, 2003, 110: 113-123
25 Kieser T, Bibb M J, Buttner M J, et al. Practical Streptomyces Genetics. Norwich, England: The John Innes Foundation, 2000. 249-250

26 Harman G E, Hayes C K, Lorito M, et al. Chitinolytic enzymes of Trichoderma harzianum: Purification of chitobiosidase and endochitinase. Phytopathology, 1993, 83: 313-318

27 Reissig J L, Storminger J L, Leloir L F. A modified colorimetric method for the estimation of $\mathrm{N}$-acetylamino sugars. J Biol Chem, 1955, 217: 959-966

28 Nampoothiri K M, Baijua T V, Sandhya C, et al. Process optimization for antifungal chitinase production by Trichoderma harzianum. Process Biochem, 2004, 39: 1583-1590

29 Kim K J, Yang Y J, Kim J G. Purification and characterization of chitinase from Streptomyces sp. M-20. J Biochem Mol Biol, 2003, 36: 185-189

30 Li Y Y, Tang J, Fu K H, et al. Construction of transgenic Trichoderma koningi with chit42 of Metarhizium anisopliae and analysis of its activity against the Asian corn borer. J Environ Sci Heal B, 2012, 47: 622-630

31 Nawani N N, Kapadnis B P. Optimization of chitinase production using statistics based experimental designs. Process Biochem, 2005, 40: 651-660

32 Noack D, Geuther R, Tonew M, et al. Expression and secretion of interferon- $\alpha 1$ by Streptomyces lividans: Use of staphylokinase signals and amplification of a neo gene. Gene, 1988, 68: 53-62

33 Bender E, Koller K P, Engels J W. Secretory synthesis of human interleukin-2 by Streptomyces lividans. Gene, 1990, 86: 227-232

Open Access This article is distributed under the terms of the Creative Commons Attribution License which permits any use, distribution, and reproduction in any medium, provided the original author(s) and source are credited. 